\title{
BMJ Open Changes in QTc interval after hydroxychloroquine therapy in patients with COVID-19 infection: a large, retrospective, multicentre cohort study
}

\author{
Moutaz El Kadri (D) ,1,2,3 Omar Al Falasi, , ${ }^{1,2}$ Rizwan Ahmed, ${ }^{1,2,3}$ Ahlam Al Awadhi, ${ }^{1,2}$ \\ Zainab Altaha, ${ }^{1,2}$ Amany Hillis, ${ }^{1,2}$ Basheer Panikkaveetil,, ${ }^{1,2}$ Sara Abdalla, ${ }^{1,2}$ \\ Honey Ansel Benette, ${ }^{1,2}$ Adhba Almubarak, ${ }^{1,2}$ Mohammed Saifuddin, ${ }^{1}$ \\ Yousef Alattar, ${ }^{1,2}$ Abderrahim Oulhaj (D) , ${ }^{3,4}$ Salem AlKaabi ${ }^{1,2}$
}

To cite: El Kadri M, Al Falasi 0, Ahmed R, et al. Changes in QTC interval after hydroxychloroquine therapy in patients with COVID-19 infection: a large, retrospective, multicentre cohort study. BMJ Open 2022;12:e051579. doi:10.1136/ bmjopen-2021-051579

- Prepublication history and additional supplemental material for this paper are available online. To view these files, please visit the journal online (http://dx.doi.org/10.1136/ bmjopen-2021-051579).

Received 23 March 2021 Accepted 18 January 2022

\section{Check for updates}

(C) Author(s) (or their employer(s)) 2022. Re-use permitted under CC BY-NC. No commercial re-use. See rights and permissions. Published by BMJ.

${ }^{1}$ Cardiology Department, Sheikh Khalifa Medical City, Abu Dhabi, UAE

${ }^{2}$ Cardiology Department, Sheikh Shakhbout Medical City, Abu Dhabi, UAE

${ }^{3}$ College of Medicine and Health Sciences, Khalifa University, Abu Dhabi, UAE

${ }^{4}$ Institute of Public Health, College of Medicine and Health Sciences, United Arab Emirates University, Al Ain, UAE

Correspondence to Dr Moutaz El Kadri; mkadri@seha.ae

\section{ABSTRACT}

Objective To evaluate the extent of hydroxychloroquineinduced corrected QT (QTC) prolongation and its relation to COVID-19 infection severity and incidence of polymorphic ventricular arrhythmias and sudden arrhythmic deaths.

Design A large-scale cohort study with retrospective analysis of baseline and on-therapy QT interval corrected using Bazett and Fridericia formulas.

Setting A multicentre study involving eight secondary and tertiary care hospitals of the Abu Dhabi Health Services Company (SEHA), United Arab Emirates.

Participants 2014 patients consecutively admitted with PCR-confirmed SARS-CoV-2 infection between 1 March 2020 and 1 June 2020.

Interventions Treatment with hydroxychloroquine alone or in combination with azithromycin for at least 24 hours and with a baseline ECG and at least one ECG after 24 hours of therapy.

Main outcome measures Maximal QTC interval prolongation and its relationship to clinical severity, polymorphic ventricular tachycardia and sudden arrhythmic death while on treatment.

Results The baseline QTc (Bazett) $_{\text {was }} 427.6 \pm 25.4 \mathrm{~ms}$ and the maximum $\mathrm{QTC}_{(\mathrm{Bazett})}$ during treatment was $439.2 \pm 30.4$ $\mathrm{ms}(\mathrm{p}<0.001)$. Severe $Q T \mathrm{~T}$ prolongation (QTc $\geq 500 \mathrm{~ms})$ was observed in $1.7 \%-3.3 \%$ of patients (Fridericia and Bazett, respectively). There were no cases of polymorphic ventricular arrhythmia or hydroxychloroquine-related arrhythmic death. QTc prolongation was more pronounced in combination therapy compared with hydroxychloroquine alone $(22.2 \mathrm{~ms}$ vs $11.0 \mathrm{~ms}, \mathrm{p}<0.001)$ and in patients with higher COVID-19 clinical severity (asymptomatic: 428.4 $\pm 25.4 \mathrm{~ms}$, severe COVID-19 infection: $452.7 \pm 35.7$ $\mathrm{ms}, \mathrm{p}<0.001$ ). The overall in-hospital mortality was $3.97 \%$ and deceased patients had longer on-therapy QTC $_{(\text {Bazett }}$ than survivors $(459.8 \pm 21.4 \mathrm{~ms}$ vs $438.4 \pm 29.9 \mathrm{~ms}$, $\mathrm{p}<0.001)$.

Conclusions The incidence of severe QTc prolongation with hydroxychloroquine was low and not associated with ventricular arrhythmia. The safety concerns surrounding the use of hydroxychloroquine may have been overestimated; however, caution should be exercised when
Strengths and limitations of this study

- This is the largest multicentre study to date with paired ECG data examining the effects of hydroxychloroquine on QTc prolongation.

- The study explores the link between clinical disease severity and QTC interval prolongation.

- The study population included patients with different clinical severity levels; hence, the effects of hydroxychloroquine on QTC in our study are more applicable to a wider population.

- The retrospective design of the study, the absence of a control group and the strong male preponderance are limitations to this study which was performed during the first wave of the COVID-19 pandemic.

using hydroxychloroquine in patients with risk factors for QT prolongation.

\section{INTRODUCTION}

The COVID-19 pandemic brought unprecedented diagnostic and therapeutic challenges to the world. Until a proven disease-specific treatment is available, repurposing of available drugs is among the few options available to reduce mortality and morbidity. ${ }^{1}$

Hydroxychloroquine (HY) is a commonly used antimalarial agent frequently prescribed for rheumatoid arthritis and systemic lupus erythematosus (SLE). Azithromycin (AZ) is a macrolide antibiotic with well-described anti-inflammatory and immunomodulatory properties. ${ }^{2}$ The antiviral efficacy of $\mathrm{HY}$ against SARS-CoV-2 in some in vitro studies ${ }^{34}$ along with favourable outcomes observed in few small-scale human studies ${ }^{56}$ led to widescale use of $\mathrm{HY} / \mathrm{AZ}$ combination early in the pandemic. ${ }^{7}$ Several subsequent studies, however, did not corroborate the clinical efficacy of these drugs ${ }^{8-11}$; on the contrary, 
possible adverse cardiovascular effects were reported, casting serious doubts on the rationale for using these drugs in patients with COVID-19. ${ }^{12-14}$

Since both HY and AZ are known to prolong QT interval, their use alone or in combination has been the subject of intense debate. ${ }^{15-17}$ Such concerns are even more valid in critically ill patients with COVID-19 who often have concomitant myocardial injury. ${ }^{18} 19$ While most studies reported QTc prolongation with these drugs, the magnitude of this prolongation and its impact on adverse cardiac outcomes such as sudden cardiac death and torsade de pointes $(\mathrm{TdP})$ were variable between different studies. ${ }^{20-27}$ For example, the incidence of extreme QTc prolongation (a marker of sudden cardiac death) varied between $2.7 \%$ and $36 \%$ depending on the study. ${ }^{17} 25$ Small sample size and differences in infection severity are among the plausible explanations for the observed discrepancy between published reports. While the use of HY to treat COVID-19 has largely been abandoned, safety concerns regarding its effect on QTc may potentially affect its use even within traditional indications such as SLE and malaria. This highlights the need for a large clinical study to clarify the effect of these medications on QT interval. ${ }^{18} 192228$ This retrospective multicentre study in a large cohort of patients with COVID-19 investigates the effect of HY therapy on QTc prolongation and any related ventricular arrhythmias or sudden arrhythmic deaths.

\section{METHODS \\ Patients}

We identified all patients with confirmed SARS-CoV-2 infection consecutively admitted to eight hospitals of Abu Dhabi Health Services Company (SEHA) between 1 March 2020 and 1 June 2020 who received HY monotherapy or HY/AZ combination therapy as part of their treatment. COVID-19 testing was performed using reverse transcription-PCR assay. A detailed retrospective chart review was performed by a team of cardiologists to assess baseline characteristics, pneumonia clinical severity and adverse events. Only patients with a baseline premedication ECG as well as a postmedication ECG recorded no earlier than 24 hours after commencing treatment were included in the analysis. Patients receiving HY for less than 24 hours or having follow-up ECG recorded within the first 24 hours of therapy or after discontinuation of therapy were excluded from analysis.

\section{Therapy regimen}

$\mathrm{HY}$ and $\mathrm{AZ}$ were given routinely to patients admitted with COVID-19 infection in the early days of the pandemic as part of the local COVID-19 treatment protocol. HY was administered orally at a dose of $400 \mathrm{mg}$ twice for the first day (loading dose), followed by $200 \mathrm{mg}$ two times per day. Patients on HY/AZ therapy also received AZ at a daily dose of $500 \mathrm{mg}$. As per institution protocol, the duration of therapy was $5-7$ days, but the final decision was left to the discretion of the treating physician.

\section{QT measurements}

ECG measurements were performed on a computer screen with digital callipers. Uncorrected QT and RR intervals were measured independently by two senior electrophysiologists and any discrepancy was resolved by agreement with a third electrophysiologist. The QT interval was calculated using the tangent method $^{29}$ and the longest QT interval of all leads was recorded according to the guidelines. ${ }^{30}$ The QT interval was reported daily (where available) for the first 5 days of treatment. The QT interval reported on day 5 was for the maximum QT interval on any ECG performed after day 4 while the patient was still on HY treatment. In patients with wide QRS (>120 ms) due to bundle branch block or paced rhythm, the QT interval was corrected using the formula QT-(QRS-120). ${ }^{31}$ QT intervals were ratecorrected with the Bazett formula $\left(\mathrm{QTc}_{(\text {Bazett }}\right)$. We also reported QTc using the Fridericia formula $\left(\mathrm{QTc}_{\text {(Fridericia) }}\right)$, since the Bazett formula is prone to overcorrection at higher heart rates. ${ }^{32}$

\section{Outcomes}

The primary outcome of interest was maximal QTc interval prolongation while on treatment. Severe QTc prolongation was defined as QTc $\geq 500 \mathrm{~ms}$ or an increase of $\geq 60 \mathrm{~ms}$ in QTc from the baseline value. ${ }^{33}$ The main secondary outcomes were $\mathrm{TdP} /$ polymorphic ventricular tachycardia (VT) and sudden arrhythmic death.

\section{Statistical analysis}

Baseline characteristics were summarised using descriptive statistics, including mean and SD for continuous measures and frequency tables for categorical variables. Categorical variables were compared using the $\chi^{2}$ or Fisher's exact test and continuous variables using the unpaired t-test or its non-parametric version (Wilcoxon rank-sum test), if the assumption of normality was not met. The paired t-test was used for the main analysis when comparing QTc intervals between baseline and different time points.

We also carried out a series of multiple linear regression models to investigate the association between mortality and severity of COVID-19 from one side and QTc prolongation from another side. In these models, the worst QTc was considered as the dependent variable and was regressed against each of the main independent variables (ie, mortality and severity of COVID-19), adjusting for available potential confounders such as age, body mass index (BMI), gender and comorbidity. All statistical tests were two-sided and $\mathrm{p}<0.05$ was considered statistically significant. Statistical analysis was conducted using $\mathrm{R}$ V.3.6.1 software (R Core Team, 2013).

\section{Patient and public involvement}

Patients and the public were not involved in the design, conduct or reporting of this research in view of its retrospective nature. 


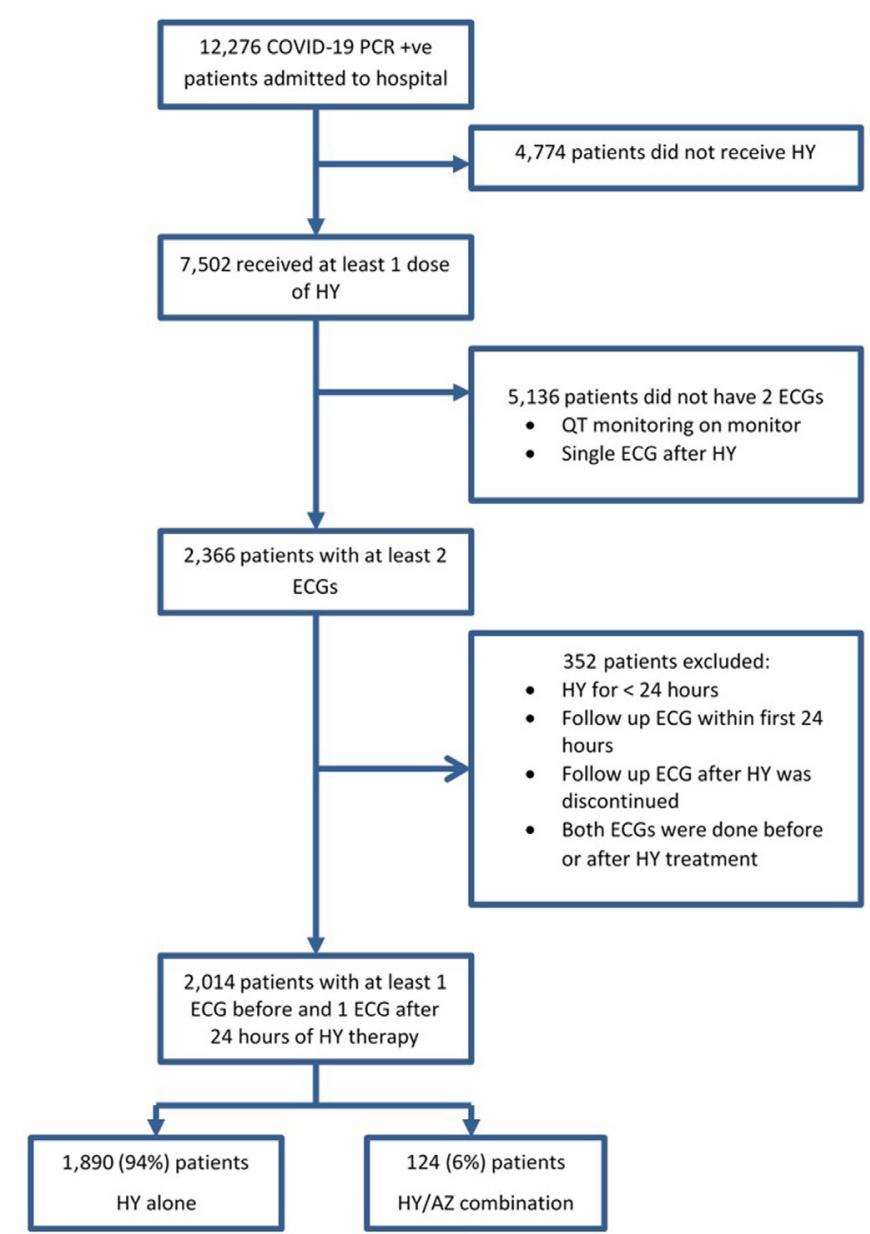

Figure 1 Flow chart of study participants included in the analysis. AZ, azithromycin; HY, hydroxychloroquine.

\section{RESULTS}

During the study period, a total of 12276 patients with COVID-19 were admitted to our medical centres and 7502 of them received at least one dose of HY. Of these, 5136 patients had an ECG performed only after HY therapy or had continuous QTc monitoring. There were 2366 patients with at least two ECGs performed during the admission. We excluded a further 352 patients for not meeting other inclusion criteria, as defined in the Patients section. The final analysis involved 2014 patients, of whom $1890(94 \%)$ received HY monotherapy and 124 $(6 \%)$ received $\mathrm{HY} / \mathrm{AZ}$ combination therapy (figure 1$)$.

The average age of patients was $46.8 \pm 12.6$ years and the majority were male $(85.8 \%)$. The average length of hospital stay (LOS) was $9.4 \pm 8.6$ days (six patients were still in hospital at the time of analysis), and the mean duration of HY treatment was 6.4 \pm 2.4 days. The LOS and duration of $\mathrm{HY}$ treatment were longer in the $\mathrm{HY} / \mathrm{AZ}$ group than in the HY group. Overall, $36.5 \%$ of the patients were diabetic, with no specific preponderance to any group. Patients with hypertension were more likely to be found in the HY group; there was no difference in the prevalence of chronic kidney disease, cancer, lung disease, structural heart disease, dialysis or liver disease in the study groups. In total, $49(2.4 \%)$ patients were immunocompromised and the prevalence of such patients was higher in the $\mathrm{HY} / \mathrm{AZ}$ group. Of all patients, $50(2.5 \%)$ were asymptomatic, and $772(38.3 \%), 736(36.5 \%)$ and $456(22.6 \%)$ had mild, moderate and severe clinical severity, respectively. The HY/AZ group had more severely infected patients compared with the HY group (41.9\% vs $21.4 \%)$. Patients requiring admission to intensive care unit (ICU), mechanical ventilation, inotropic support or dialysis were also more prevalent in the HY/AZ group (table 1).

The overall in-hospital mortality was $3.97 \% \quad(80$ patients), which was relatively higher in the $\mathrm{HY} / \mathrm{AZ}$ group $(5.65 \%)$ than in the HY group $(3.86 \%)$; however, the difference did not reach statistical significance $(p=0.46)$. Only eight patients $(10 \%)$ were receiving $\mathrm{HY}$ at the time of death. Sudden death was observed in only four patients (5\%), all of whom were still receiving $\mathrm{HY}$ at the time of death. Cardiac arrest was due to asystole in two patients $(2.5 \%)$ and pulseless electrical activity (PEA) in the other two patients $(2.5 \%)$. In all remaining cases, a clear clinical deterioration in the hours preceding cardiorespiratory arrest was observed. Cardiac arrest was commonly caused by bradycardia and asystole (55 of 80 patients, $68.7 \%$ ). PEA was the cause of cardiac arrest in 23 patients $(28.8 \%)$, whereas monomorphic VT was observed only in 2 patients $(2.5 \%)$, neither of whom was on HY at the time of death. There were no cases of polymorphic VT or TdP.

A modest but statistically significant QTc prolongation was observed during treatment. The mean $\mathrm{QTc}_{\text {(Bazett) }}$ increased by $11.6 \mathrm{~ms}$ from $427.6 \pm 25.4 \mathrm{~ms}$ at baseline to $439.2 \pm 30.4 \mathrm{~ms}$ during therapy $(\mathrm{p}<0.001)$. QTc (Fridericia) had lower absolute numerical values compared with QTc ${ }_{(\text {Bazett })}$; however, the pattern of QTc increase was similar (baseline: 402.8 $\pm 23.2 \mathrm{~ms}, \mathrm{HY}: 419.5 \pm 28.2 \mathrm{~ms}, \mathrm{p}<0.001)$. The higher values with $\mathrm{QTC}_{(\text {Bazett })}$ were largely due to overcorrection during tachycardia since 441 (21.9\%) patients had heart rate $\geq 100$ beats per minute at baseline. Almost one-third of the patients had a decrease in QTc while on treatment, primarily due to the resolution of tachycardia with supportive treatment; hence, this effect was more apparent with $\mathrm{QTc}_{\text {(Bazett) }}$. QTc $\geq 500 \mathrm{~ms}$ and $\Delta$ QTc $\geq 60 \mathrm{~ms}$ were observed in $3.3 \%$ and $4.5 \%$ of patients, respectively, using Bazett formula, and in $1.7 \%$ and $5.5 \%$ of patients, respectively, using Fridericia formula (figure 2).

The temporal changes in QTc interval during HY therapy revealed a daily increase in both $\mathrm{QTc}_{\text {(Bazett) }}$ and QTc $_{\text {(Fridericia) }}$ until day 3, after which the relative increase in QTc was less prominent (figure 3). In the $\mathrm{HY} / \mathrm{AZ}$ combination therapy group, QTc (Bazett) $_{\text {increased from } 431 \pm 25}$ $\mathrm{ms}$ to $451 \pm 36 \mathrm{~ms}$, whereas in the HY monotherapy group the value increased only to $438 \pm 30 \mathrm{~ms}$ from a baseline value of $427 \pm 25 \mathrm{~ms}$. A similar trend was observed in QTc Fridericia), with an increase of $28.8 \mathrm{~ms}$ and $16.0 \mathrm{~ms}$ in the $\mathrm{HY} / \mathrm{AZ}$ and HY groups, respectively (figure 4).

Patients with more severe COVID-19 infection had greater QTc prolongation while on HY treatment. The

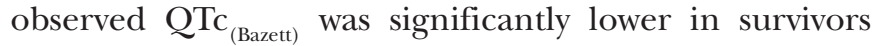
than it was in the deceased $(438.4 \pm 29.9 \mathrm{~ms}$ vs $459.8 \pm 21.4$ ms, $\mathrm{p}<0.001)$. A similar trend was also observed using 
Table 1 Baseline characteristics, risk factors and clinical course of patients

\begin{tabular}{|c|c|c|c|c|}
\hline & $\begin{array}{l}\text { Total } \\
2014(100 \%) \\
\end{array}$ & $\begin{array}{l}\text { HY only } \\
1890(94 \%)\end{array}$ & $\begin{array}{l}\text { HY/AZ } \\
124(6 \%)\end{array}$ & P value* \\
\hline \multicolumn{5}{|l|}{ Baseline characteristics } \\
\hline Age, mean $( \pm S D)$ & $46.8( \pm 12.6)$ & $47.0( \pm 12.6)$ & $43.8( \pm 12.2)$ & 0.005 \\
\hline Male sex, n (\%) & $1727(85.7)$ & $1619(85.6)$ & $108(87.1)$ & 0.756 \\
\hline \multicolumn{5}{|l|}{ Ethnicity, n (\%) } \\
\hline African & $15(0.7)$ & $15(0.8)$ & $0(0.0)$ & 0.686 \\
\hline Arab & 367 (18.2) & $342(18.1)$ & $25(20.3)$ & \\
\hline Asian & $1612(80.2)$ & $1515(80.3)$ & $97(78.3)$ & \\
\hline Caucasian & $11(0.5)$ & $10(0.5)$ & $1(0.8)$ & \\
\hline Other & $7(0.4)$ & $6(0.3)$ & $1(0.8)$ & \\
\hline Length of stay (days), mean $( \pm S D)$ & $9.4( \pm 8.6)$ & $9.0( \pm 8.3)$ & $15.2( \pm 10.7)$ & $<0.001$ \\
\hline Length of $\mathrm{HY}$ treatment (days), mean $( \pm \mathrm{SD})$ & $6.4( \pm 2.3)$ & $6.3( \pm 2.3)$ & $7.6( \pm 2.7)$ & $<0.001$ \\
\hline \multicolumn{5}{|l|}{ Clinical risk factors } \\
\hline $\mathrm{BMI}$, mean $( \pm \mathrm{SD})$ & $27.6( \pm 5.0)$ & $27.7( \pm 5.1)$ & $26.4( \pm 4.6)$ & 0.003 \\
\hline \multicolumn{5}{|l|}{ BMI categories, $\mathrm{n}(\%)$} \\
\hline$<25$ & $593(33.3)$ & 549 (32.9) & 44 (39.3) & 0.057 \\
\hline $25-30$ & 711 (39.9) & $662(39.7)$ & $49(43.7)$ & \\
\hline $30-40$ & 425 (23.9) & $406(24.3)$ & $19(17.0)$ & \\
\hline$>40$ & $51(2.9)$ & $51(3.1)$ & $0(0.0)$ & \\
\hline \multicolumn{5}{|l|}{ Smoking status, $\mathrm{n}(\%)$} \\
\hline Current smoker & $109(5.4)$ & $107(5.7)$ & $2(1.6)$ & 0.028 \\
\hline Former smoker & $74(3.7)$ & $73(3.9)$ & $1(0.8)$ & \\
\hline Non-smoker & $1831(90.9)$ & $1710(90.4)$ & $121(97.6)$ & \\
\hline Diabetes, n (\%) & $736(36.5)$ & $695(36.8)$ & $41(33.1)$ & 0.463 \\
\hline Hypertension, n (\%) & $786(39.0)$ & 749 (39.6) & $37(29.8)$ & 0.038 \\
\hline CKD, n (\%) & $141(7.0)$ & $132(6.9)$ & $9(7.3)$ & 1.000 \\
\hline Cancer, n (\%) & $49(2.5)$ & $45(2.4)$ & $4(3.2)$ & 0.771 \\
\hline Lung disease, $n(\%)$ & $118(5.9)$ & $113(6.0)$ & $5(4.0)$ & 0.486 \\
\hline Structural heart disease, $\mathrm{n}(\%)$ & $155(7.7)$ & $150(7.9)$ & $5(4.0)$ & 0.160 \\
\hline Liver disease, $\mathrm{n}(\%)$ & $15(0.7)$ & $14(0.7)$ & $1(0.8)$ & 1.000 \\
\hline Immunosuppression, n (\%) & $49(2.4)$ & $42(2.2)$ & $7(5.6)$ & 0.036 \\
\hline \multicolumn{5}{|l|}{ Clinical course } \\
\hline \multicolumn{5}{|l|}{ Clinical severity, $n(\%)$} \\
\hline Asymptomatic & $50(2.5)$ & $46(2.4)$ & $4(3.2)$ & $<0.001$ \\
\hline Mild & $772(38.3)$ & $731(38.7)$ & $41(33.1)$ & \\
\hline Moderate & $736(36.6)$ & $709(37.5)$ & 27 (21.8) & \\
\hline Severe & $456(22.6)$ & $404(21.4)$ & $52(41.9)$ & \\
\hline \multicolumn{5}{|l|}{ CXR findings, $\mathrm{n}(\%)$} \\
\hline Consolidation & $1390(69.0)$ & $1294(68.5)$ & $96(77.4)$ & 0.031 \\
\hline No consolidation & $251(12.5)$ & $235(12.4)$ & $16(12.9)$ & \\
\hline CXR not performed & $373(18.5)$ & $361(19.1)$ & $12(9.7)$ & \\
\hline \multicolumn{5}{|l|}{ Lung CT findings, $\mathrm{n}(\%)$} \\
\hline Normal & $80(4.0)$ & $73(3.7)$ & $7(5.6)$ & $<0.001$ \\
\hline Mild changes & $523(26.0)$ & $496(26.3)$ & $27(21.8)$ & \\
\hline Moderate changes & $785(39.0)$ & $758(40.2)$ & $27(21.8)$ & \\
\hline
\end{tabular}


Table 1 Continued

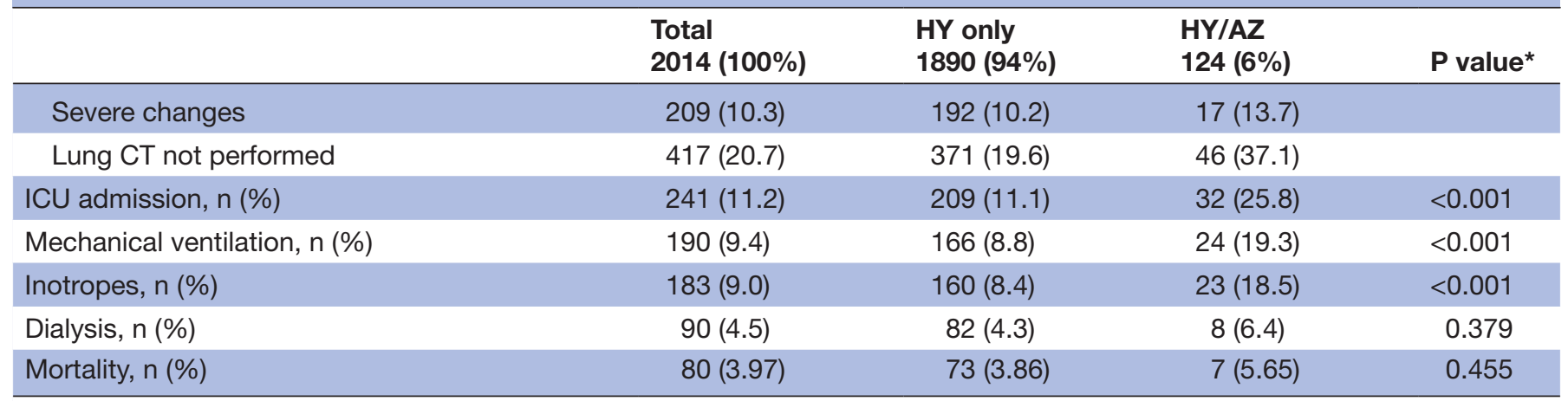

${ }^{*}$ Continuous variables were summarised using t-test, while discrete variables were summarised using $\chi^{2}$ test.

AZ, azithromycin; BMI, body mass index; CKD, chronic kidney disease; CXR, chest X-ray; HY, hydroxychloroquine; ICU, intensive care unit.

$\mathrm{QTc}_{(\text {Fridericia) }}$. There was a systematic increase in $\mathrm{QTc}_{(\mathrm{Bazett})}$ and QTc $_{\text {(Fridericia) }}$ values with increasing clinical infection severity. The mean values of $\mathrm{QTc}_{\text {(Bazett) }}$ in asymptomatic, mild, moderate and severely infected patients were $428.4 \pm 25.4 \mathrm{~ms}, 432.3 \pm 27.2 \mathrm{~ms}, 438.9 \pm 27.5 \mathrm{~ms}$ and $452.7 \pm 35.7 \mathrm{~ms}$, respectively $(\mathrm{p}<0.001)$; $\mathrm{QTc}_{\text {(Fridericia) }}$ also exhibited a similar pattern (figure 5). The associations between QTc (Bazett) and $_{\text {QTc }}$ (Fridericia) $_{\text {from one side and }}$ mortality and severity of COVID-19 from another side
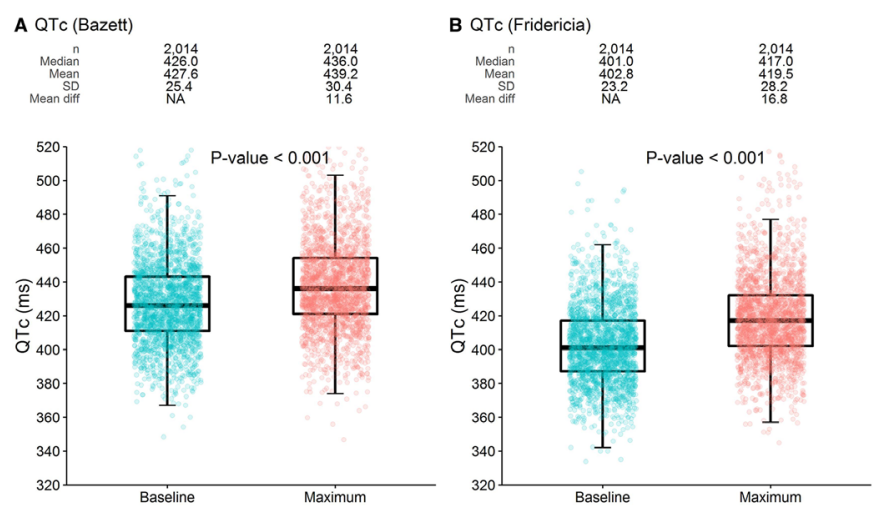

C QTC (Bazett)

D QTC (Fridericia)
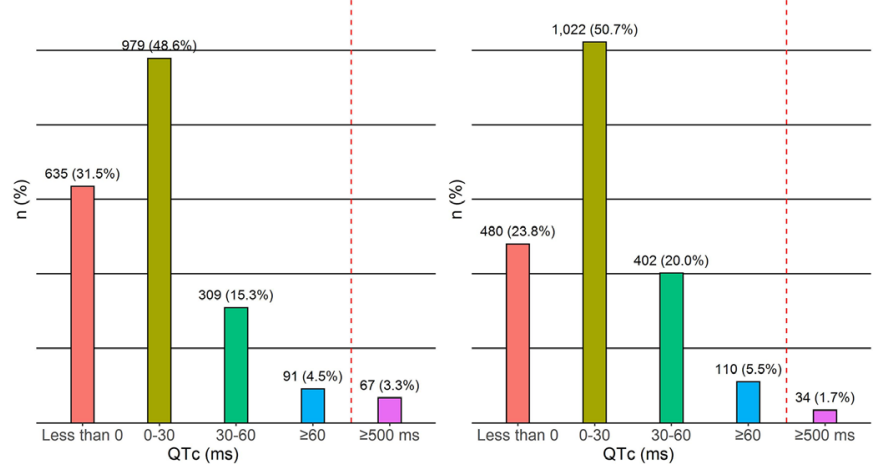

Figure 2 Changes in QTc interval in patients treated with hydroxychloroquine (with or without azithromycin). (A, B) Baseline and peak QTc interval using Bazett and Fridericia formulas, respectively. (C, D) Distribution of patients stratified by degree of QTc change using Bazett and Fridericia formulas, respectively. QTc, corrected QT; NA, not applicable. were still statistically significant when multiple linear regression models adjusting for age, gender, BMI and comorbidity were used. The details of these adjusted analyses are reported in online supplemental tables 1-4.

\section{DISCUSSION}

This large cohort study with paired ECG data suggests a clinically modest but statistically significant QTc prolongation after $\mathrm{HY}$ or $\mathrm{HY} / \mathrm{AZ}$ therapy. Like other studies, ${ }^{21} 34$ QTc prolongation was evident from the first day of therapy and showed an increasing daily trend suggestive of a possible cumulative effect. Notably, however, QTc prolongation was less marked than most other studies on patients with COVID-19 ${ }^{17}$ and was more in line with previous large-scale studies in patients with rheumatological diseases. ${ }^{26} 35$ Studies on patients with COVID-19 reported a highly variable degree of QTc prolongation, which is unsurprising given the differences in sample size, demographics and clinical severity in these studies. These shortcomings were largely overcome in our study by virtue of its large sample size and covering different clinical severities.

In our cohort, the peak average QTc was higher in HY/ AZ combination therapy than in HY monotherapy. This

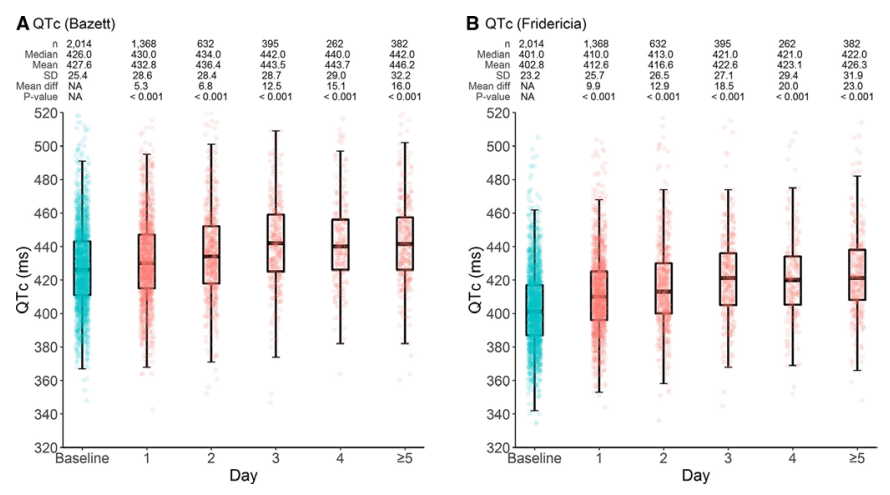

Figure 3 Baseline and daily QTc interval change in patients treated with hydroxychloroquine (with or without azithromycin) using (A) Bazett and (B) Fridericia formulas, respectively. QTc, corrected QT; NA, not applicable. 


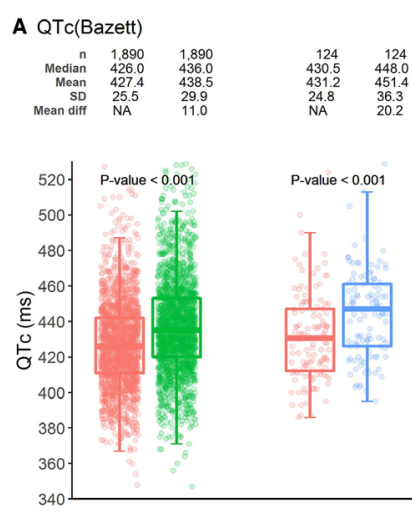

追 Baseline 追 $\underset{(\mathrm{Max})}{\mathrm{HCQ} \text { only }}$ 追 $\underset{(\mathrm{Max})}{\mathrm{HCQ}+\mathrm{AZITH}}$
B QTc(Fridericia)
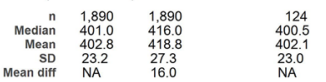
124
425.0
430.9
38.3
28.8

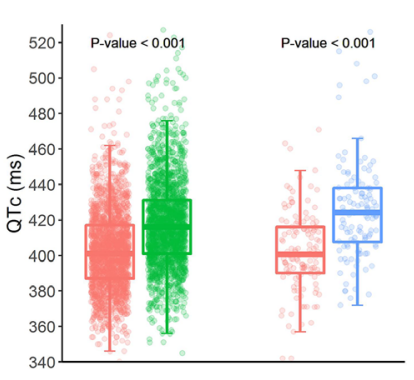

追 Baseline 追 $\underset{(\mathrm{Max})}{\mathrm{HCQ} \text { only }}$ 追 $\underset{(\mathrm{Max})}{\mathrm{HCQ}+\mathrm{AZITH}}$
Figure 4 Baseline and maximal QTc measurements in patients treated with hydroxychloroquine (HCQ) alone or in association with azithromycin (AZITH) using (A) Bazett and (B) Fridericia formulas, respectively. QTc, corrected QT; NA, not applicable.

was expected since both drugs are known to prolong QTc interval. ${ }^{36}$ In the combination therapy group, there was a $20.2 \mathrm{~ms}$ increase in $\mathrm{QTc}_{\text {(Bazett) }}$ in the HY/AZ group and $11.0 \mathrm{~ms}$ in the HY group from their respective baseline values $(p<0.001)$. This QTc prolongation in the combination group is broadly similar to the $20-30 \mathrm{~ms}$ increase reported by several other investigators. ${ }^{17} 192434$ In our study, patients receiving combination therapy were more likely to have higher clinical COVID-19 severity and longer hospital stay. The need for ICU admission, mechanical ventilation and inotropic support was also
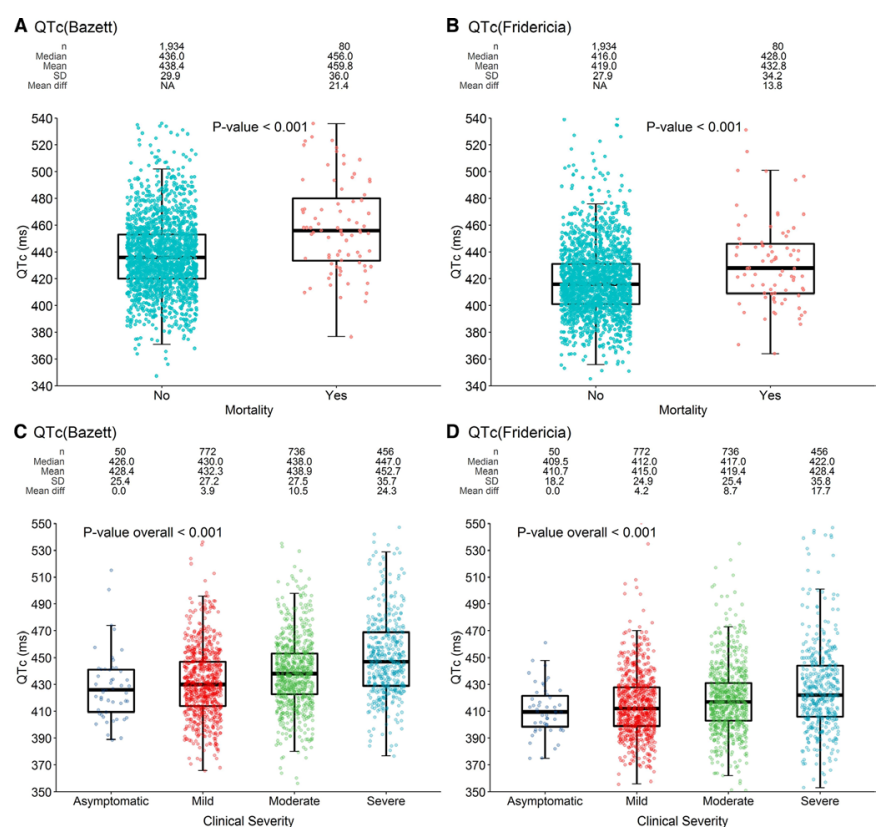

Figure 5 Relationship between QTc and mortality and disease severity. (A, B) Maximal QTc interval in survivors and deceased patients (Bazett and Fridericia formulas, respectively). Distribution of maximal QTc intervals stratified by clinical severity of COVID-19 infection is shown in (C) and (D) using Bazett and Fridericia formulas, respectively. QTc, corrected QT; NA, not applicable. more likely in this group, reflecting a more turbulent clinical course. The frequent use of combination therapy in higher severity cases likely reflects the need for a more aggressive therapeutic approach in these patients.

The incidence of critical QTc prolongation was relatively low in our cohort compared with other studies. ${ }^{19}$ Hooks $e t a t^{26}$ reported a similar low incidence of $1.5 \%$ in rheumatological patients on HY therapy. In contrast, the incidence of severe QTc prolongation in literature from the COVID-19 era ranged between $11 \%$ and $36 \%$, with most patients being treated with $\mathrm{HY} / \mathrm{AZ}$ combination. ${ }^{172124}{ }^{36}$ Such a variance can be attributed to the differences in the clinical severity and the demographics of the patients included in these studies and our younger cohort. $^{1721}$

The overall mortality in our study was $3.97 \%$, with no cases of polymorphic VT, TdP or sudden death due to ventricular arrhythmia. The mortality rate in our study was significantly lower than the $21 \%-27 \%$ mortality rate reported in other studies. ${ }^{11} 2437$ There are several possible explanations for this observation. In contrast to other studies, our study population was significantly younger and HY was administered liberally irrespective of clinical severity (ie, use not restricted to severe cases). Another favourable factor in our case was that the healthcare system coped well with the pandemic and was never overwhelmed; therefore, optimal care continued to be provided to all admitted patients. Finally, differences in the virulence of the virus strain may have been a contributing factor in explaining the differences in fatality rates observed in different parts of the world, although more research is needed to establish such a factor.

Our study highlights the effects of COVID-19 infection severity on QTc duration. Overall, QTc prolongation during treatment was more pronounced in patients with higher clinical severity. A stepwise increase in QTc interval during HY treatment was proportional to the increase in clinical severity from asymptotic to severe. Indeed, patients with the highest severity leading to fatality had the most prolonged QTc in the whole study $(459.8 \pm 36.0 \mathrm{~ms}$ (Bazett), 432.8 $\pm 34.2 \mathrm{~ms}$ (Fridericia)). Electrolyte abnormalities, myocardial injury, renal impairment and polypharmacy are all more common in patients with severe infection, possibly compounding QTc prolongation. ${ }^{38} 39$ Our observations highlight the multifactorial nature of QTc prolongation. The simultaneous presence of several QT-prolonging factors (such as drugs, genetic predisposition, electrolyte imbalance, severe illness) often has a synergistic effect, occasionally leading to marked QTc prolongation. ${ }^{40}$

To account for the impact of tachycardia frequently observed in patients with COVID-19 on QTc calculations, we reported QTc measurements using both Bazett and Fridericia formulas. Indeed, in our study, almost a quarter of the patients were admitted with sinus tachycardia. The Fridericia formula probably offers better rate correction in this setting, a finding also observed by Vandenberk et $a l .{ }^{32}$ Our results suggest that, although 
there was a noticeable difference in the calculated QTc values by these two approaches, both showed a similar trend.

The demographics and patient characteristics in our study reflect the social structure and workforce distribution in United Arab Emirates. The majority of patients in this study were Asian men, relatively young, but with a high prevalence of diabetes and hypertension. Many of these expatriate workers live in shared accommodation, possibly explaining the higher representation of Asian men among SARS-CoV-2-infected patients in our study.

The main strength of this study is that it is the largest multicentre study to date with paired ECG data examining the effects of HY on QTc prolongation. Another strength of the study is the inclusion of patients with different clinical severity levels. Therefore, the effects of HY on QTc in our study are more applicable to a wider population compared with previous studies predominantly recruiting Caucasian patients with severe infection. Our study also reports QTc values by two methods and therefore factors in the effect of heart rate on QTc measurements. One of the major limitations of the study is its retrospective design and the absence of a control group. ECG data collection from a drug-free control group was not possible due to the liberal use of HY in most patients with COVID-19 in our hospitals at that time. In addition, it was difficult to justify performing non-clinically indicated ECGs in a control group at a time when healthcare resources were already overstretched and it was vital to protect staff by reducing unnecessary exposure to patients with COVID-19. However, the lack of a control group was compensated for by the paired nature of our measurements reducing the intersubject variability. In addition, since AZ was used only as an additional therapy to HY and not as monotherapy, we do not have an AZ-only group; hence, we cannot comment on its isolated effect on QTc. Furthermore, there may be a degree of selection bias with ECGs potentially being recorded in patients deemed to be at higher risk of QT prolongation. In addition, due to the large sample size and retrospective nature of the study, it was not possible to confirm whether patients were receiving other QT-prolonging drugs during HY therapy. However, the institutional protocol for HY therapy mandated regular monitoring of drug interactions by clinical pharmacists, thereby limiting the impact of this factor. Moreover, our data are mainly from patients with COVID-19 infection, with a strong male preponderance, possibly limiting the generalisability of the study findings to women and patients without COVID-19. Finally, our results may not be relevant anymore to the treatment of patients with COVID-19 given the rapid decline in the use of $\mathrm{HY}$ and $\mathrm{AZ}$ in this group. However, the fact that our population was younger and with lower clinical severity compared with other studies may make our results more relevant during HY treatment for other conditions such as malaria and SLE.

\section{CONCLUSION}

Among patients with COVID-19 prescribed HY alone or in combination with AZ, there was a modest QTc prolongation. The incidence of extreme QTc prolongation was low and not associated with any major druginduced cardiovascular events. Although the use of HY to treat COVID-19 has largely been abandoned, it remains widely indicated to treat other conditions. Thus, when HY is used appropriately and with adequate cardiac monitoring, it remains a safe drug with only a trivial risk of significant adverse cardiac events. Caution should, however, be exercised with the concomitant use of HY with other QT-prolonging drugs or with very sick patients.

Acknowledgements The authors would like to thank Editverse (www.editverse. com) for their help with manuscript editing. The authors also acknowledge the huge commitment and dedication of our healthcare workers as well as the intense suffering and pain endured by our patients and their families during these difficult times.

Contributors MEK contributed to the study design, literature search, data collection, drafting the manuscript, and data analysis and interpretation. OAF and SAK contributed to the study design, critical review of the manuscript, and data collection and interpretation. RA contributed to data collection and interpretation, and drafting and critical review of the manuscript. $A 0$ contributed to statistical analysis, drafting the figures and critical review of the manuscript. AAA, ZA, AH, BP, SA, HAB, AA, MS and YA contributed to data collection and editing and review of the manuscript. The corresponding author attests that all listed authors meet the authorship criteria and that no others meeting the criteria have been omitted. MEK accepts responsibility as guarantor.

Funding The authors have not declared a specific grant for this research from any funding agency in the public, commercial or not-for-profit sectors.

Competing interests MEK has received honoraria for consultancy for Medtronic, Abbott, Pfizer, Bayer, Saja and Boehringer Ingelheim. SAK has received honoraria for consultancy for Medtronic, Bayer, Pfizer and Saja. OAF has received honoraria for consultancy for Medtronic. None of these interests are related to this article. All other authors declare no competing interests.

Patient consent for publication Not required.

Ethics approval This study involves human participants and was approved by the National Emirates Institutional Review Board for COVID-19 research (DOH/ CVDC/2020/831) and was conducted in accordance with the ethical standards laid down in the 1964 Declaration of Helsinki and its later amendments. The requirement for informed consent was waived for this retrospective analysis.

Provenance and peer review Not commissioned; externally peer reviewed.

Data availability statement Data are available upon reasonable request. Individual participant data will be made available on request, directed to the corresponding author (MEK). Requests will be assessed for scientific rigour before being granted. After approval of a proposal, data will be anonymised and securely transferred. A data sharing agreement may be required.

Supplemental material This content has been supplied by the author(s). It has not been vetted by BMJ Publishing Group Limited (BMJ) and may not have been peer-reviewed. Any opinions or recommendations discussed are solely those of the author(s) and are not endorsed by BMJ. BMJ disclaims all liability and responsibility arising from any reliance placed on the content. Where the content includes any translated material, BMJ does not warrant the accuracy and reliability of the translations (including but not limited to local regulations, clinical guidelines, terminology, drug names and drug dosages), and is not responsible for any error and/or omissions arising from translation and adaptation or otherwise.

Open access This is an open access article distributed in accordance with the Creative Commons Attribution Non Commercial (CC BY-NC 4.0) license, which permits others to distribute, remix, adapt, build upon this work non-commercially, and license their derivative works on different terms, provided the original work is properly cited, appropriate credit is given, any changes made indicated, and the use is non-commercial. See: http://creativecommons.org/licenses/by-nc/4.0/. 


\section{ORCID iDs}

Moutaz El Kadri http://orcid.org/0000-0003-3761-5546

Abderrahim Oulhaj http://orcid.org/0000-0002-5330-904X

\section{REFERENCES}

1 Guy RK, DiPaola RS, Romanelli F, et al. Rapid repurposing of drugs for COVID-19. Science 2020;368:829-30.

2 Zimmermann P, Ziesenitz VC, Curtis N, et al. The immunomodulatory effects of Macrolides-A systematic review of the underlying mechanisms. Front Immunol 2018;9:302.

3 Yao X, Ye F, Zhang M, et al. In vitro antiviral activity and projection of optimized dosing design of hydroxychloroquine for the treatment of severe acute respiratory syndrome coronavirus 2 (SARS-CoV-2). Clin Infect Dis 2020;71:732-9.

4 Liu J, Cao R, Xu M, et al. Hydroxychloroquine, a less toxic derivative of chloroquine, is effective in inhibiting SARS-CoV-2 infection in vitro. Cell Discov 2020;6:16.

5 Gautret P, Lagier J-C, Parola P, et al. Hydroxychloroquine and azithromycin as a treatment of COVID-19: results of an openlabel non-randomized clinical trial. Int $J$ Antimicrob Agents 2020;56:105949.

6 Chen Z, Hu J, Zhang Z. Efficacy of hydroxychloroquine in patients with COVID-19: results of a randomized clinical trial. medRxiv 2020:2020.

7 Gautret P, Lagier J-C, Parola P, et al. Hydroxychloroquine and azithromycin as a treatment of COVID-19: results of an openlabel non-randomized clinical trial. Int $J$ Antimicrob Agents 2020;56:105949.

8 Molina JM, Delaugerre C, Le Goff J, et al. No evidence of rapid antiviral clearance or clinical benefit with the combination of hydroxychloroquine and azithromycin in patients with severe COVID-19 infection. Med Mal Infect 2020;50:384.

9 Rosenberg ES, Dufort EM, Udo T, et al. Association of treatment with hydroxychloroquine or azithromycin with in-hospital mortality in patients with COVID-19 in New York state. JAMA 2020;323:2493-502.

10 Furtado RHM, Berwanger O, Fonseca HA, et al. Azithromycin in addition to standard of care versus standard of care alone in the treatment of patients admitted to the hospital with severe COVID-19 in Brazil (coalition II): a randomised clinical trial. Lancet 2020;396:959-67.

11 Geleris J, Sun Y, Platt J, et al. Observational study of hydroxychloroquine in hospitalized patients with Covid-19. N Engl J Med 2020;382:2411-8.

12 Fiolet T, Guihur A, Rebeaud ME. Effect of hydroxychloroquine with or without azithromycin on the mortality of coronavirus disease 2019 (COVID-19) patients: a systematic review and meta-analysis. Clin Microbiol Infect 2020.

13 Ferner RE, Aronson JK. Chloroquine and hydroxychloroquine in covid-19. BMJ 2020;369:m1432.

14 Pastick KA, Okafor EC, Wang F, et al. Review: hydroxychloroquine and chloroquine for treatment of SARS-CoV-2 (COVID-19). Open Forum Infect Dis 2020;7:ofaa130.

15 Svanström H, Pasternak B, Hviid A. Use of azithromycin and death from cardiovascular causes. N Engl J Med 2013;368:1704-12.

16 Oren O, Yang EH, Gluckman TJ, et al. Use of chloroquine and hydroxychloroquine in COVID-19 and cardiovascular implications: understanding safety discrepancies to improve interpretation and design of clinical trials. Circ Arrhythm Electrophysiol 2020;13:e008688.

17 Bessière F, Roccia H, Delinière A, et al. Assessment of QT intervals in a case series of patients with coronavirus disease 2019 (COVID-19) infection treated with hydroxychloroquine alone or in combination with azithromycin in an intensive care unit. JAMA Cardiol 2020;5:1067-9.

18 Moschini L, Loffi M, Regazzoni V. Effects on QT interval of hydroxychloroquine associated with ritonavir/darunavir or azithromycin in patients with SARS-CoV-2 infection. Heart Vessels 2020:1-6.

19 Mercuro NJ, Yen CF, Shim DJ, et al. Risk of QT interval prolongation associated with use of hydroxychloroquine with or without concomitant azithromycin among hospitalized patients testing positive for coronavirus disease 2019 (COVID-19). JAMA Cardiol 2020;5:1036-41.

20 Cavalcanti AB, Zampieri FG, Rosa RG. Hydroxychloroquine with or without azithromycin in mild-to-moderate Covid-19. N Engl J Med 2020.

21 Chorin E, Wadhwani L, Magnani S, et al. Qt interval prolongation and torsade de pointes in patients with COVID-19 treated with hydroxychloroquine/azithromycin. Heart Rhythm 2020;17:1425-33.

22 Cipriani A, Zorzi A, Ceccato D, et al. Arrhythmic profile and 24-hour QT interval variability in COVID-19 patients treated with hydroxychloroquine and azithromycin. Int J Cardiol 2020;316:280-4.

23 Saleh M, Gabriels J, Chang D. Safely administering potential QTC prolonging therapy across a large healthcare system in the COVID-19 era. Circulation: Arrhythmia and Electrophysiology, 2020.

24 O'Connell TF, Bradley CJ, Abbas AE. Hydroxychloroquine/ Azithromycin therapy and QT prolongation in hospitalized patients with COVID-19. JACC: Clinical Electrophysiology 2020;1261.

25 Bun S-S, Taghji P, Courjon J, et al. QT interval prolongation under Hydroxychloroquine/Azithromycin association for inpatients with SARS-CoV-2 lower respiratory tract infection. Clin Pharmacol Ther 2020;108:1090-7.

26 Hooks M, Bart B, Vardeny O, et al. Effects of hydroxychloroquine treatment on QT interval. Heart Rhythm 2020;17:1930-5.

27 Monzani A, Genoni G, Scopinaro A, et al. QTc evaluation in COVID-19 patients treated with chloroquine/hydroxychloroquine. Eur J Clin Invest 2020;50:e13258.

28 Das RR, Jaiswal N, Dev N, et al. Efficacy and safety of anti-malarial drugs (chloroquine and Hydroxy-Chloroquine) in treatment of COVID-19 infection: a systematic review and meta-analysis. Front Med 2020;7:482.

29 Postema PG, Wilde AAM. The measurement of the QT interval. Curr Cardiol Rev 2014;10:287-94.

30 Rautaharju PM, Surawicz B, Gettes LS, et al. AHA/ACCF/HRS recommendations for the standardization and interpretation of the electrocardiogram: Part IV: the ST segment, T and U waves, and the QT interval: a scientific statement from the American heart association electrocardiography and arrhythmias Committee, Council on clinical cardiology; the American College of cardiology Foundation; and the heart rhythm Society. endorsed by the International Society for computerized Electrocardiology. J Am Coll Cardiol 2009;53:982-91.

31 Patel P, Borovskiy Y, Deo R. QTCC, a novel method for correcting QT interval for QRS duration, predicts all-cause mortality. J Am Coll Cardiol 2015;65:A336.

32 Vandenberk B, Vandael E, Robyns T, et al. Which QT correction formulae to use for QT monitoring? J Am Heart Assoc 2016;5. doi:10.1161/JAHA.116.003264. [Epub ahead of print: 1706 2016].

33 Drew BJ, Ackerman MJ, Funk M, et al. Prevention of torsade de pointes in hospital settings: a scientific statement from the American heart association and the American College of cardiology Foundation. Circulation 2010;121:1047-60.

34 Chorin E, Dai M, Shulman E, et al. The QT interval in patients with COVID-19 treated with hydroxychloroquine and azithromycin. Nat Med 2020;26:808-9.

35 Costedoat-Chalumeau N, Hulot J-S, Amoura Z, et al. Heart conduction disorders related to antimalarials toxicity: an analysis of electrocardiograms in 85 patients treated with hydroxychloroquine for connective tissue diseases. Rheumatology 2007;46:808-10.

36 Ramireddy A, Chugh $\mathrm{H}$, Reinier K, et al. Experience with hydroxychloroquine and azithromycin in the coronavirus disease 2019 pandemic: implications for QT interval monitoring. J Am Heart Assoc 2020;9:e017144.

37 Horby $\mathrm{P}$, Mafham M, et al. Effect of hydroxychloroquine in hospitalized patients with Covid-19. N Engl J Med 2020

38 van Noord C, Eijgelsheim M, Stricker BHC. Drug- and nondrug-associated QT interval prolongation. Br J Clin Pharmacol 2010;70:16-23.

39 Farré N, Mojón D, Llagostera M, et al. Prolonged QT interval in SARS-CoV-2 infection: prevalence and prognosis. J Clin Med 2020;9. doi:10.3390/jcm9092712. [Epub ahead of print: 2108 2020].

40 Vandael E, Vandenberk B, Vandenberghe J, et al. Risk factors for QTc-prolongation: systematic review of the evidence. Int J Clin Pharm 2017;39:16-25. 